\title{
Fuzzy Based Optimization for Power Management in Wireless Sensor Networks
}

\author{
K.Sheela Sobana Rani \\ Ass.Prof., SNS College of Technology, \\ Coimbatore.
}

\author{
N.Devarajan, PhD. \\ Prof., Government College of Technology
}

\begin{abstract}
In Wireless Sensor Networks (WSN), sensor node deployment is essential for maximizing the coverage and detection probabilities. But the existing optimization solution suffers from limited energy storage, node death, increased network traffic etc. To solve these issues, we propose a fuzzy based optimization model for power management in wireless sensor networks. The objectives considered in the paper include maximizing network coverage, connectivity, network lifetime and minimizing traffic load. A fuzzy rules table is constructed with the input parameters such as node degree, link quality, residual energy and traffic rate. Depending upon the outcome of the fuzzy logic, the nodes are categorized into good, normal and bad. After the initial deployment of good nodes, the SLEEP and WAKEUP Procedure is applied to maximize the lifetime of wireless sensor networks. By putting nodes to sleep when there are no events, the energy consumption of the sensor nodes can be significantly reduced. In SLEEP and WAKEUP Procedure, asynchronous type is used; it allows each node in the wireless sensor network to set its own SLEEP and WAKEUP schedule independently in order to save energy. Simulation result shows that the proposed Procedure provides maximization of the lifetime of Wireless Sensor Network (WSN).
\end{abstract}

\section{INTRODUCTION}

Wireless sensor networks (WSN) helps in sensing or monitoring physical or environment conditions since the network has distributed autonomous nodes. Sensor nodes consist of one or more sensors, a radio transceiver, a microcontroller and an energy source. Currently WSN has fascinated more number of research interests which is due to potential applications in many areas ranging from environmental observation, natural habitat monitoring, medical, industry and military applications. Searching and developing potentials are essential for formation of multiple mobile sensor nodes in the sensor network. The entire monitoring environment needs to be covered by the mobile sensor nodes using the searching potentials of the sensor network. For the region where concentrated monitoring is essential the sensor nodes are joined using the developing potentials of the sensor network. A novel deployment strategy is required to maximize both the potentials of the sensor networks. Depending upon the coverage of each point in the sensing field, the sensors provide better QoS. [1]The maximum network coverage needs to be achieved by effectively deploying the sensors in an appropriate manner. Energy efficient protocols are aimed at minimizing the energy consumption during network activities. However, a large amount of energy is consumed by node components (CPU, radio, etc.) even if they are idle. Energy or power management schemes are thus used for switching off node components that are not temporarily needed. Several energy conservation schemes have been proposed. They are mainly aimed at minimizing the energy consumption of the communication subsystem. [2]There are two main approaches to energy conservation in-network processing and power saving through duty cycling. In-network processing consists in reducing the number of information to be transmitted by means of compression or aggregation techniques.

\section{Fuzzy based Node selection \\ 2.1 Overview}

In the proposed model, the following objectives are considered for each sensor:
1)
To maximize network coverage
To maximize connectivity
3)
To maximize network lifetime
4)
To minimize traffic load

Initially for each sensor, the node degree, link quality, residual energy and traffic rate are estimated in order to ensure the coverage, connectivity, network lifetime and traffic load, respectively. These parameters are then passed on to a Fuzzy Logic Engine to form the fuzzy rules. Based on the outcome of the fuzzy rules, the nodes are categorized into 3 levels namely good, normal and bad. Then multi-objective PSO based optimization technique is applied for normal and bad type of nodes to refine the above objectives keeping the levell (good) nodes as reference points. So at the end of the PSO iteration process, each bad and normal level nodes are connected to atleast one good node. Since fuzzy logic is used for decision making, the accuracy of the objective functions will be high.

\subsection{Estimation of Sensor Parameters}

\subsubsection{Estimation of Node Degree $(\mathrm{Nd})$}

Assume $\mathrm{K}$ random uniformly distributed nodes in a sensor network. Each node uses the same wireless communication module with a fixed unidirectional communication range. Nodes within the communication range $\mathrm{C}$ may communicate with each other and are denoted as 1-hop-neighbors. [6]One node is taken as central node (Node zero).

Node density $\mathrm{Nd}$ in the scenario is strongly linked to the node degree $\operatorname{deg}(\mathrm{K})$, which is the number of neighbors.

$$
N d=\frac{\operatorname{deg}(K)}{\pi \cdot c^{2}}
$$


When shortest communication path or the shortest hop count is n, two nodes are considered as n-hop neighbors. The distance between a node pair is taken as a for the n-hop neighbors and the observed probability is $\operatorname{Pn}(\mathrm{a})$. The communication range $\mathrm{C}$ will be set to 1 (distance unit) as simple scaling, resulting in a unit-disc-graph.

\subsubsection{Estimation of Link Quality (LQ)}

Link quality is estimated using packet delivery ratio and channel quality.

(i) Packet delivery ratio (Pd)

The Packet Reception Ratio (PRR) is computed as the ratio of the number of successfully received packets (Ns) to the number of transmitted packets (Nt), for each window of $\mathrm{k}$ received packets.

$\mathrm{PRR}=\mathrm{Ns} / \mathrm{Nt}$

WMEWMA is then given by the following:

$$
\begin{aligned}
\operatorname{Pd} & =\operatorname{WMEWMA}(\lambda, \mathrm{K}) \\
& =\lambda \times \mathrm{WMEWMA}+(1-\lambda) \times \mathrm{PRR}
\end{aligned}
$$

where $\lambda €[0 . .1]$ controls the smoothness.

\section{(ii) Channel Quality (Cq)}

Received signal (Rs) is obtained by sampling the RSSI at the packet reception and Noise floor $(\mathrm{F})$ can be obtained from the RSSI sample just after the packet reception. The signal to noise ratio can be obtained by subtracting noise floor $(\mathrm{N})$ from the received signal $(\mathrm{S})$ which is given by

$$
\mathrm{SNR}=\mathrm{Rs}-\mathrm{F}
$$

SNR value is averaged over $\mathrm{k}$ received packets to get $\mathrm{Cq}$ for channel assesment

Link quality metric is the summation of both the packet delivery ratio and channel quality.

$$
\mathrm{LQ}=\mathrm{A} \times \mathrm{Pd}+\mathrm{B} \times \mathrm{Cq}
$$

\subsubsection{Estimation of Residual Energy $(R E)$}

The residual energy $(\mathrm{R})$ is calculated as the difference between initial energy and transmitting energy.

$$
R E=\eta-\eta_{t}
$$

\subsubsection{Estimation of Traffic rate (Ld)}

(i) Estimation of Number of Monitored sources
A distance-from-source label will be put on exploratory data packets, and nodes consider the sum of monitored sources with counter distance quotient as V Metric.

$$
V_{S}=\frac{1}{p} \sum_{i=1}^{p}\left(\frac{1}{q_{i}}\right)
$$

(ii) Estimation of Value of Traffic in Each Node The value of traffic in each node is denoted by the metric Vt. This metric shows the passing traffic from one node during one phase of algorithm execution. The following equation shows the calculation for Vt metric.

$V_{t}=1-\frac{U_{m}}{U_{\text {max }}}$

where $U_{\max }=\sum_{j=1}^{p} U_{m_{j}}$ and $\mathrm{Um}$ indicates the number of packets sent during one phase by one node, $\mathrm{U}_{\max }$ is the max maximum number of packets sent during one phase by one source.

\section{(iii) Estimation of Traffic Load at Each Node}

The traffic load in each node is denoted by the metric Ld

$L d=\mathrm{V} s \times V t$

\subsection{Categorization of Nodes using Fuzzy logic}

2.3.1 Fuzzy Logic

Rule definition: A membership function characterizes a fuzzy set A which can be implemented by fuzzy conditional statements. If antecedent is true to some degree of membership in a fuzzy statement, then consequent also proves to be true to the same degree.

Rule structure: If antecedent then consequent

Rule: The output is benign when variable 1 and 2 are low and other variable 3 is high. Otherwise the output is malignant.

\subsubsection{Fuzzification}

In this work, the fuzzy if-then rules consider the parameters: Node degree, link quality, Residual energy and traffic load in order to categorize the nodes for deployment. The resulting possibilities are Good (G), Normal(N) and Bad(B). Here the inputs can take 2 values Low and High. Hence the total number of outputs in this case is $24=16$. The selection criterion is such that a node should have higher node degree, higher link quality, higher residual energy and lower traffic load. 


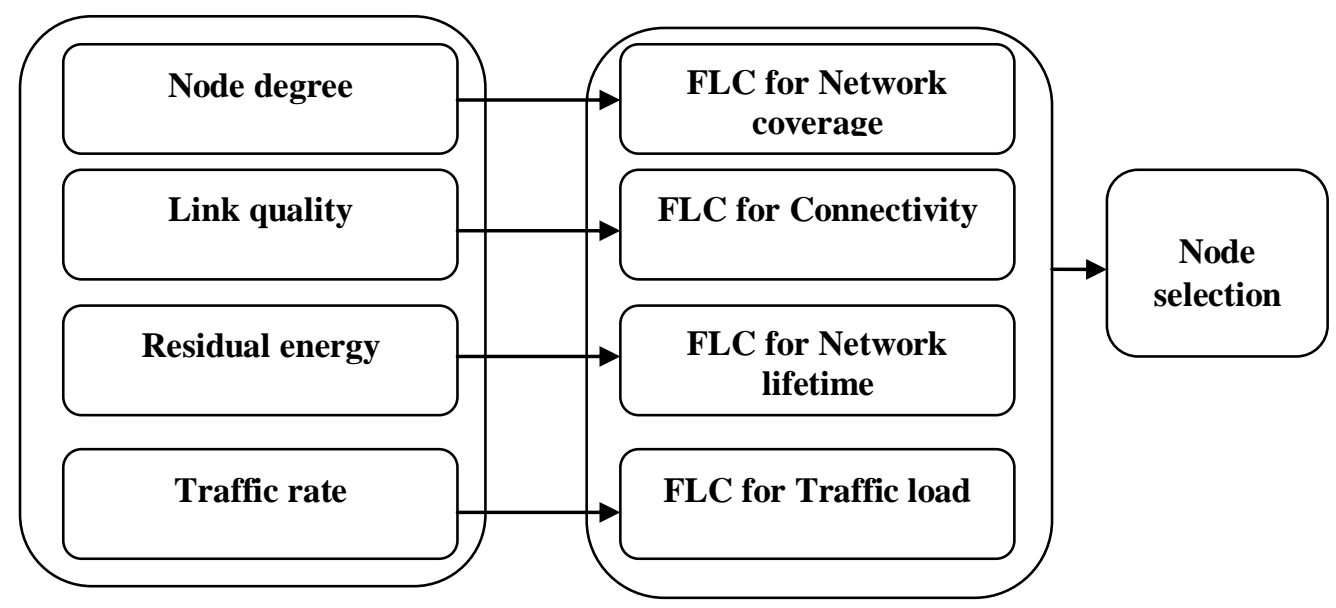

Figure 2.1: Fuzzy logic system

The final decision is made on the basis of the output of the intersection of the corresponding members of the fuzzy sets of the four parameters. In order to deploy the sensor nodes efficiently we have to maximize the node degree, link quality and the Residual energy. The load factor must be reduced. In this fuzzy table we consider the output is good when any three conditions are satisfied. The output is taken as normal when any two conditions are satisfied. Satisfaction of no or one parameter leads to bad output value. Based upon this, the fuzzy rules table 1 is created.

\subsubsection{Defuzzification}

The techniques used in the defuzzification of the fuzzified values includes: centroid average method, max centre method, mean of maxima, smallest of maximum and largest of maximum[7].

Center of Area (COA) In this method the output is given as a crispy value. This value depends upon the output membership function of the center of gravity.

$$
U_{0}=\frac{\int w \mu(w) d w}{\int \mu(w) d w}
$$

\section{ASYNCHRONOUS SLEEPS AND WAKEUP PROCEDURE}

Asynchronous schemes avoid the tight synchronization among network nodes required by scheduled rendezvous schemes. They allow each node to wakeup independently of the others by guaranteeing that neighbors always have overlapped active periods within a specified number of cycles.

In Figure 3.1, considering sensor networks where static sensor nodes are randomly located in a given region and are placed at one hop / Multi hop distance. Considering the traffic in the network to be light, transmissions are collision free. Taking one of the deployed nodes as Gateway Node / Data collection center for transmitting the message from home network to nearby network and applying SLEEP and WAKEUP Procedure for the remaining Node in the network. Since $\mathrm{Ng}$ Node act as the Gateway Node / Data Collection Centre it must be always active, so the energy utilized by $\mathrm{Ng}$ (Gateway Node) is high when compared to other nodes in the same network. [3] Initialize which node wants to transmit the data to gate way, the transmitter node may placed at one hop / multi hop distance, if its placed at multi hop it should transmit via intermediate nodes it can get a chance to transmit immediately / wait in $T_{\text {sav }}$ mode till the intermediate node gets active. Single hop and multi hop can be both used for transmitting the data to the Gateway Node / Data Collection Center. 


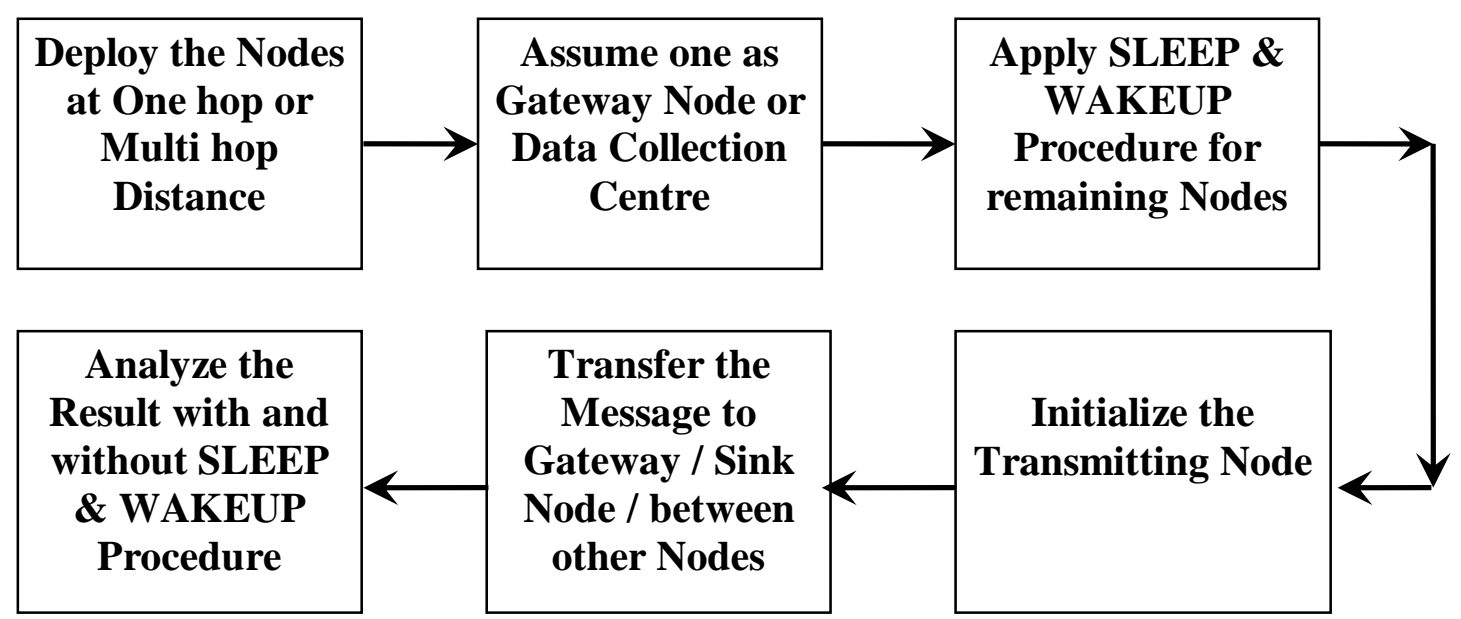

Figure 3.1 Block diagram for the proposed method

As in Figure 3.2, nine Nodes are deployed including Gateway Node at one hop distance; $\mathrm{Ng}$ is the Gateway Node and the Gateway Node is active through out the network lifetime. The scenario we used here is all-to-one environment where all Nodes periodically report data to a sink (Gateway Node / Data Collection Centre).

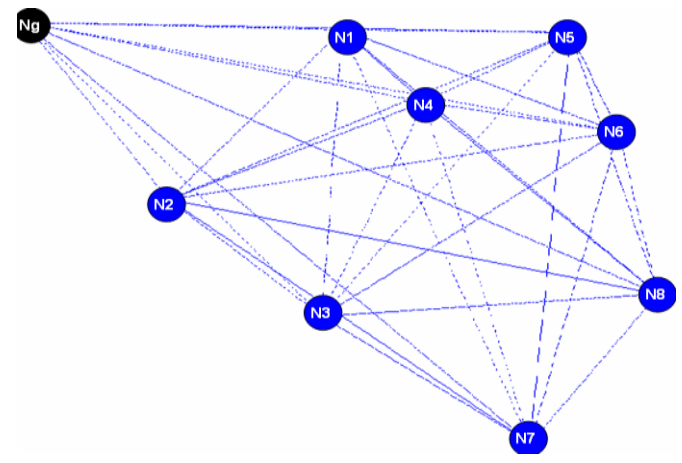

Figure 3.2 Topology of a network with eight sensors at one hop distance

Considering sensor networks where static sensor nodes are randomly located in a given region, the traffic in the network is light, and hence transmissions are collision free. [4]The average rate at which packets are flowed over the link from node $i$ to j. Defining $i$ as the neighbour set of node $j$ with

$\mathrm{j}\{=\mathrm{i}$ node $\mathrm{j}$ is in RF range of node $\mathrm{i} \neq \mathrm{i}$ otherwise

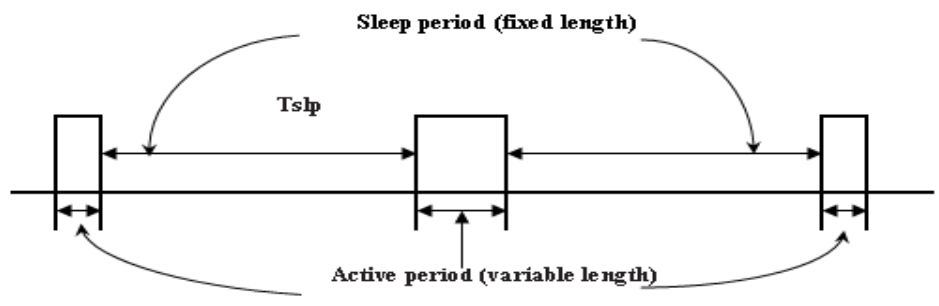

Figure 3.3 Asynchronous sleep scheduling mechanism

A sensor node is either in an active mode / a sleep mode according to its sleep schedule, as depicted in Figure 3.3.
In the sleep mode, a node is turned off and no power is consumed. Every time, a node i goes into the sleep mode, 
it sleeps for a period $\boldsymbol{T}_{s l p}$ of time before waking up. The sleep time of a node is fixed, but different nodes have different sleep times due to different traffic and battery distributions. A node wakes up and enters an active mode from time to time to see if it has any packets to transmit or if there are any other nodes attempting to transmit to it. An active period can be further categorized as an idle listening slot, a data transmission slot, or a data reception slot. In an idle listening slot,

+ Ptx Tdata + Prx Tpr

if the node neither transmits nor receives data packets within this active period. An active period is said to be a data transmission slot if the node transmits a packet in this slot[5]. Likewise, an active period is a data reception slot if the node receives a packet in this slot.

\subsection{Transmitter Energy}

To compute the energy consumptions of a node during an active period, this could be an idle listening slot, a data transmission slot, or a data receiving slot. In a data transmission slot, the average energy consumption for node $i$ to transmit one packet to node $j$ is

$$
\begin{array}{ll}
\mathrm{E}_{\mathrm{tx}}=\left(\mathrm{E}_{\mathrm{rf}}+\mathrm{P}_{\mathrm{rx}}+\mathrm{T}_{\text {det }}\right) & \mathrm{A} \\
-\mathrm{E}_{\mathrm{rf}}+\left(\mathrm{E}_{\mathrm{rf}}+\left(\mathrm{P}_{\mathrm{tx}}+\mathrm{P}_{\mathrm{rx}}\right) \mathrm{T}_{\text {pre }}\right) & B
\end{array}
$$

$$
\mathrm{X} \underset{2 \mathrm{Tpre})) /(\operatorname{Trf}+2 \mathrm{Tpre}+\mathrm{Tsav})+2\} \mathrm{C}(12)}{\left(\left(\mathrm{T}_{\mathrm{slp}} / 2\right)-\left(2 \mathrm{~T}_{\mathrm{pre}}+\mathrm{T}_{\mathrm{sav}}+\mathrm{T}_{\mathrm{rf}}+\right.\right.}
$$

Where, $\mathrm{A}$ is the energy cost due to RF initialization and channel detection, B \& C are the energy cost to transmit the RTS packets until an RTS packet is acknowledged, while D represents the energy cost to transmit the data packet and receive the ACK packet.

\subsection{Receiver Energy}

The energy consumption for a node to receive a packet is calculated as

$$
\begin{aligned}
& \text { Erx }=[\text { Erf }+\operatorname{Prx}(\operatorname{tdet} / 2)]+[\text { Prx Tdata }]+[2 \operatorname{Ptx} \\
& \text { Tpre }]
\end{aligned}
$$

where the first term on the right hand side is the energy cost to initialize RF circuit and the average energy cost to detect the RF channel before desired RTS is received, the second term is the energy cost to receive the data packet, while the third term is the energy cost to transmit the CTS and ACK packets. Furthermore, the energy cost due to idle listening is

$$
\mathrm{E}_{\mathrm{det}}=\mathrm{E}_{\mathrm{rf}}+\mathrm{P}_{\mathrm{rx}}\left(\mathrm{T}_{\mathrm{det}}-\mathrm{T}_{\mathrm{rf}}\right)
$$

\section{SIMULATION RESULTS}

During simulation two types of Procedures have been analyzed i.e. with and without SLEEP and WAKEUP Procedure for the nodes placed at one hop and multi hop distance.The nodes are working at constant RF transmission power. All nodes have the same initial battery capacity. The network life times obtained by two Procedures are compared

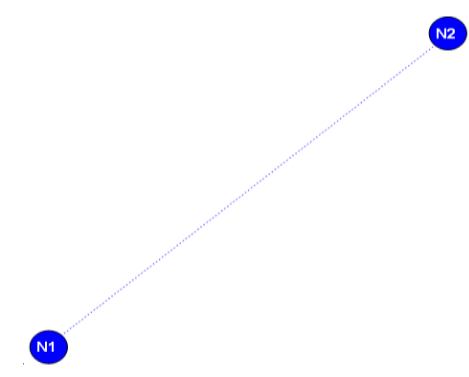

\section{Figure 4.1 Two nodes deployed at one hop}

The nodes are placed in 100x100 square areas, randomly for one hop distance. From the Figure 4.1 analyzing that two nodes are placed at one hop distance, assumption here is nodes can transmit the data to all the nodes, not in particular(Gateway

\section{Figure 4.2 Three nodes deployed at one hop}

Node). Condition for the scenario described by Figure 4.1 is $\mathrm{N} 1$ transmitting the data to $\mathrm{N} 2$ and the channel listening time is $.008756 \mathrm{sec}$, initial energy is 1000 ; here the two nodes are active during simulation. 
Table 4.1 Energy comparison when two nodes are active

\begin{tabular}{|c|c|c|}
\hline \multicolumn{3}{|c|}{ Energy remaining in Nodes after simulation } \\
\hline \multicolumn{3}{|c|}{ Channel listening mode is .008756 sec } \\
\hline Procedure & Transmitter (Joules) & Receiver (Joules) \\
\hline SLEEP and WAKEUP & 999.9991 & 999.9993 \\
\hline Without SLEEP and WAKEUP & 999.9991 & 999.9993 \\
\hline
\end{tabular}

Table 4.2 Energy comparison when Transmitter is active and Receiver is in sleep mode

\begin{tabular}{|c|c|c|}
\hline \multicolumn{3}{|c|}{ Energy remaining in Nodes after simulation } \\
\hline \multicolumn{3}{|c|}{ Channel listening mode is .008756 sec } \\
\hline Procedure & Transmitter (Joules) & Receiver (Joules) \\
\hline SLEEP and WAKEUP & 999.9990 & 999.9993 \\
\hline With out SLEEP and WAKEUP & 999.9193 & 999.9993 \\
\hline
\end{tabular}

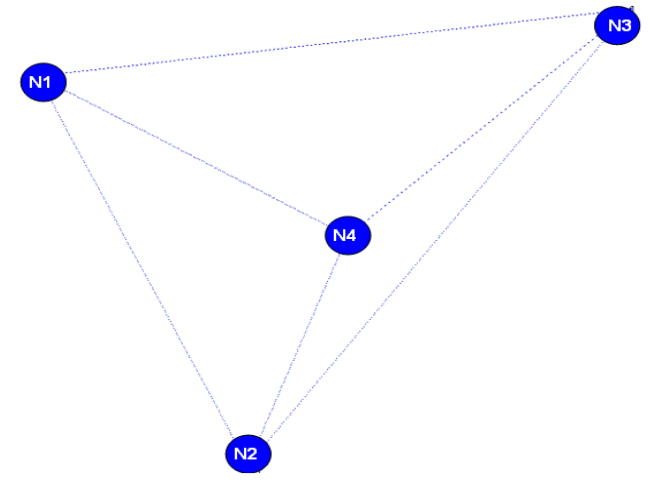

Figure 6.3 Four nodes deployed at one hop distance

Table 4.3 Energy comparison when Transmitter is in sleep mode and Receiver is in active

\begin{tabular}{|c|c|c|}
\hline \multicolumn{3}{|c|}{ Energy remaining in Nodes after simulation } \\
\hline \multicolumn{3}{|c|}{ Channel listening mode is .008756 sec } \\
\hline Procedure & Transmitter (Joules) & Receiver (Joules) \\
\hline SLEEP and WAKEUP & 999.9491 & 999.9993 \\
\hline With out SLEEP and WAKEUP & 999.9993 & 999.9993 \\
\hline
\end{tabular}

In Figure 4.3 at the time of simulation Node1 is in sleep mode, after it gets active it has some data to transmit to
Node 4. The energy remaining in Node 1 and Node 4 is listed in Table 4.3 
Table 4.4 Energy comparisons when both Transmitter and Receiver is in sleep mode

\begin{tabular}{|c|c|c|}
\hline \multicolumn{3}{|c|}{ Energy remaining in Nodes after simulation } \\
\hline \multicolumn{2}{|c|}{ Channel listening mode is .008756 sec } & Receiver (Joules) \\
\hline Procedure & Transmitter (Joules) & 999.9193 \\
\hline SLEEP and WAKEUP & 999.9490 & 999.9993 \\
\hline
\end{tabular}

Figure 4.3 show that at the time of simulation Node1 is in sleep mode, after it gets active it has some data to transmit to Node 3 . Here the Node 3 is also in sleep mode. The energy remaining in Node 1 and Node 3 is listed in Table 4.4.The Node will transmit the data to the gate way with channel listening period of $0.008756 \mathrm{sec}$, the remaining 9 minutes and $999965 \mathrm{sec}$ it moves periodically between
Channel listening and Sleep Mode for SLEEP and WAKEUP Procedure whereas without SLEEP and WAKEUP Procedure the remaining time i.e. 9 minutes and 999965 seconds the node is in channel listening mode with the receiving power of $45 \mathrm{~mW}$. The energy comparison is listed in Table 4.5.

Table 4.5 Energy comparisons when Transmitter (N1 - N8) transmitting data to Ng at single hop distance

\begin{tabular}{|c|c|c|}
\hline \multicolumn{3}{|c|}{$\begin{array}{c}\text { Energy remaining in Nodes after simulation } \\
\text { (Simulation time for the Network is 10 minutes) }\end{array}$} \\
\hline \multicolumn{2}{|c|}{ Channel listening mode is .008756 sec } \\
\hline Procedure & Transmitter (Joules) & Gateway (Joules) \\
\hline SLEEP and WAKEUP & 997.8976 & 973.5028 \\
\hline With out SLEEP and WAKEUP & 973.113 & 973.5028 \\
\hline
\end{tabular}

Energy utilized by the nodes for transmitting the data from $\mathrm{N} 1$ to $\mathrm{Ng}$, at the time of transmitting data from $\mathrm{N} 1$ i.e. in channel listening mode of N1, N5 is active so it take intermediate node as $\mathrm{N} 5$, and transmit the data to $\mathrm{Ng}$. Here N5 utilized more energy when compare to N1, because it act as intermediate node i.e. it collects the data form N1 and transmit to $\mathrm{Ng}$. The remaining nodes move periodically between channel listening and sleep mode.

\section{CONCLUSION}

Sensor Networks hold a lot of promise in applications where gathering sensing information in remote locations is required. Many factors can influence the energy consumption in wireless sensor networks. Their energyconstrained nature necessitates one to look at more energy efficient design and operation.. Depending upon the outcome of the fuzzy logic, the nodes are categorized into good, normal and bad. After the initial deployment of good nodes, the SLEEP and WAKEUP Procedure is applied to maximize the lifetime of wireless sensor networks. By putting nodes to sleep when there are no events, the energy consumption of the sensor nodes can be significantly reduced. In SLEEP and WAKEUP Procedure, asynchronous type is used; it allows each node in the wireless sensor network to set its own SLEEP and WAKEUP schedule independently in order to save energy. Simulation result shows that the proposed Procedure provides maximization of the lifetime of Wireless Sensor Network (WSN).

\section{REFERENCES}

[1] Ahmed, H. Shi, and Y. Shang, "A Survey on Network Protocols for Wireless Sensor Networks", International Conference on Information Technology: Research and Education, pp.301 - 305, August 2003.

[2] Chipcon AS, "SmartRF CC2420 Preliminary Datasheet", rev.1.2, February 2004.

[3] C. Schurgers, V. Tsiatsis, M.B. Srivastava, "STEM: Topology Management for Energy Efficient Sensor Networks", IEEE Aerospace Conference '02, March, 2002.

[4] "Energy Harvesting and Conservation", IEEE Pervasive Computing, Vol. 4, Issue 1, January-March. 2005.

[5] Feng Liu, Chi-Ying Tsui, Member, IEEE, and Ying Jun (Angela) Zhang, Member, "Joint Routing and Sleep Scheduling for Lifetime Maximization of Wireless Sensor Networks", IEEE Transactions On Wireless Communications, pp.2258-2267, July 2010.

[6] Rico Radeke and Stefan Türk "Node Degree based Improved Hop Count Weighted Centroid Localization Algorithm" Conference on Communication in Distributed Systems (KiVS'11). Editors: Norbert Luttenberger, Hagen Peters; pp. 194-199.

[7] Yigang Shi and P.C. Sen, "A New Defuzzification Method for Fuzzy Control of Power Converters" Industry Applications Conference, 2000. Conference Record of the 2000 IEEE. 